\title{
Atención de enfermería al preescolar con neumonía viral
}

\section{Yudith Felipe-de la Cruz ${ }^{1}$}

RESUMEN: La neumonía es la principal causa de morbimortalidad en niños menores de cinco años. El presente estudio expone el caso de una preescolar de dos años que presentó dificultad respiratoria, ventilando con apoyo de oxígeno por cánula binasal a tres litros con un $\mathrm{FiO} 2$ de $32 \%$ con $\mathrm{SatO} 2$ 99\%, frecuencia respiratoria de 48 por minuto, se observó tiraje intercostal, presencia de estertores a la auscultación en ambos campos pulmonares, presentó piel caliente y mejillas ruborizadas con $38.7^{\circ} \mathrm{C}$. Después de la valoración del caso se realizó la planificación de cuidados de enfermería, para ello se plantearon cuatro diagnósticos reales "Patrón respiratorio ineficaz", "Hipertermia", "Limpieza ineficaz de las vías aéreas" y "Ansiedad del niño". Se efectuó el plan de cuidados durante los primeros días de hospitalización de la preescolar usando el modelo de valoración de los 13 dominios seleccionando los aspectos positivos y negativos de la Taxonomía II de NANDA-I y la clasificación de diagnósticos, intervenciones y resultados de la Taxonomía II. Se concluyó que a través del plan de cuidados de enfermería se logró la recuperación del patrón respiratorio, la hipertermia y la limpieza ineficaz de las vías aéreas, asimismo se consiguió disminuir la ansiedad del niño.

PALABRAS CLAVE: Neumonía viral; Monitoreo fisiológico; Oxigenoterapia; Ansiedad

Citar como: Felipe Y. Atención de enfermería al preescolar con neumonía viral. CASUS. 2017; 2(1):71-79. 


\section{INTRODUCCIÓN}

Según la Organización Mundial de la Salud (OMS) la neumonía es considerada una infección respiratoria y principal causa de morbi mortalidad infantil a nivel mundial principalmente en niños menores de cinco años. Se calcula que esta enfermedad en el año 2015 fue causante del $15 \%$ de todas las muertes de esta población $(1,2)$. En el mismo año se diagnosticaron 27 de casos por cada 10000 niños menores de cinco años en el Perú a diferencia del 2014 que se diagnosticaron 20.5/10 000 (3).

Los niños preescolares son muy vulnerables a las neumonías en especial de tipo viral. Esta situación afecta el patrón respiratorio y los niveles de saturación de oxígeno. Dependiendo de la gravedad de la enfermedad respiratoria se brindará un tratamiento de soporte mediante la oxigenoterapia (4). El mismo ayudará a conservar los niveles de oxígeno dentro de los límites normales y prevenir complicaciones como la hipoxia tisular (5). La monitorización respiratoria es esencial en la atención del infante porque trata y evita complicaciones en el estado respiratorio (6). Por otro lado se evidencia que la fisioterapia respiratoria mejora el patrón respiratorio en niños con neumonía porque ayuda a remover las secreciones de las vías respiratorias bajas (7).

Es importante ofrecer una atención de enfermería óptima enfocada en la recuperación del estado fisiológico. De este modo se logrará que el niño tenga menos días de hospitalización $\mathrm{y}$ disminuir su ansiedad (8). Considerando lo anterior se planteó ejecutar las intervenciones de enfermería al preescolar de dos años con neumonía viral, priorizando y mejorando el estado respiratorio. Para la solución del caso se usó el modelo de los 13 dominios de la taxonomía II de Nursing Diagnoses Classification (NANDA)-I, intervenciones Nursing Interventions Classification (NIC) y resultados de enfermería Nursing Outcomes Classification (NOC) (9-11).

\section{PRESENTACIÓN DEL CASO}

Se presenta el caso clínico de una preescolar de dos años de edad. Ingresó por emergencia pediátrica en compañía de sus padres presentando dificultad respiratoria, taquipnea de 50 respiraciones por minuto, hipoxia, SatO2 90\%. Mediante la auscultación presentó ruidos anormales en ambos campos pulmonares. Asimismo presentó una temperatura corporal de $37.8^{\circ} \mathrm{C}$ y 148 pulsaciones rítmicas por minuto. Se le diagnosticó neumonía viral recibiendo como indicaciones médicas oxigenoterapia por cánula binasal a cuatro litros, antipiréticos condicional a la fiebre, corticoide $\mathrm{y}$ electrolitos.

\section{VALORACIÓN}

Para el estudio del caso clínico se realizó la valoración usando los dominios de la taxonomía II de NANDA-I enfocándose en el estado actual de la paciente. La preescolar presento una temperatura de $38.7^{\circ} \mathrm{C}$, una SatO2 de 99\% con apoyo de oxígeno por cánula binasal, 48 respiraciones por minuto, tiraje intercostal y estertores a la auscultación en ambos campos pulmonares. Asimismo presento ansiedad por estancia hospitalaria (ver tabla 1). 
Tabla 1. Análisis de los dominios según aspectos positivos y negativos

\begin{tabular}{|c|c|c|}
\hline Dominios & Positivos & Negativos \\
\hline Promoción de la Salud & $\begin{array}{l}\text { - Pre-escolar acompañada de su } \\
\text { madre. } \\
\text { - Mamá apoya a la enfermera y al } \\
\text { médico con el tratamiento. }\end{array}$ & - No se observa. \\
\hline Nutrición & - No se observa. & - Apetito disminuido. \\
\hline Eliminación e Intercambio & $\begin{array}{l}\text { - Orina espontáneamente. } \\
\text { - Genitales sin lesiones. }\end{array}$ & • No se observa. \\
\hline Actividad y Reposo & • Sueño conservado. & $\begin{array}{l}\text { - Frecuencia cardiaca de } 138 \\
\text { por minuto. } \\
\text { - S/ CBN SatO2 90\%. } \\
\text { - Frecuencia respiratorio } 48 \\
\text { por minuto. } \\
\text { - Tiraje intercostal. } \\
\text { - Recibe oxigenoterapia por CBN } \\
\text { a 3lts. FiO2 32\%. }\end{array}$ \\
\hline Percepción / Cognición & $\begin{array}{l}\text { - Lenguaje adecuado para su edad. } \\
\text { - Cumple órdenes. } \\
\text { - Despierta. }\end{array}$ & • No se observa. \\
\hline Autopercepción & - No se observa & $\begin{array}{l}\text { - Irritable. } \\
\text { - Llorosa. }\end{array}$ \\
\hline Rol / Relación & $\begin{array}{l}\text { - Pre-escolar vive con sus padres } \\
\text { y hermano de } 6 \text { años. }\end{array}$ & • No se observa. \\
\hline Sexualidad & $\begin{array}{l}\text { - Genitales de acuerdo a su edad } \\
\text { y sexo. } \\
\text { - Higiene adecuada. }\end{array}$ & • No se observa. \\
\hline Afrontamiento / Tolerancia al estrés & - No se observa. & $\begin{array}{l}\text { - Preescolar ansiosa por su } \\
\text { estancia hospitalaria. }\end{array}$ \\
\hline Principios Vitales & $\begin{array}{l}\text { - Religión católica. } \\
\text { - Madre refiere no tener problemas } \\
\text { con la transfusión sanguínea. }\end{array}$ & • No se observa. \\
\hline Seguridad y Protección & • No se observa. & $\begin{array}{l}\text { - Vía periférica permeable en MSI. } \\
\text { - Temperatura de } 38.7^{\circ} \mathrm{C} \text { axilar. } \\
\text { - Presenta secreciones pero no } \\
\text { abundantes. }\end{array}$ \\
\hline Confort & $\begin{array}{l}\text { - Ausencia de dolor. } \\
\text { - Reposando en cama. }\end{array}$ & • No se observa. \\
\hline Crecimiento/Desarrollo & $\begin{array}{l}\text { - Mide } 87 \mathrm{~cm} \text { de estatura } 11 \mathrm{~kg} \text {. } \\
\text { de peso. } \\
\text { - Desarrollo psicomotor adecuado. }\end{array}$ & - No se observa. \\
\hline
\end{tabular}




\section{FASE DIAGNÓSTICA}

Conforme a la valoración realizada a la preescolar de dos años se formularon los diagnósticos de enfermería de la taxonomía II de NANDA. Se priorizaron cuatro diagnósticos reales que permitieron proporcionar los cuidados de enfermería en respuesta a las necesidades fisiológicas $\mathrm{y}$ emocionales.

\section{Reales:}

-(00032) Patrón respiratorio ineficaz r/c fatiga de los músculos respiratorios e/p disnea, taquipnea, tiraje intercostal, SO2 90\% sin cánula binasal. Dominio 4, Clase 4.

-(00007) Hipertermia r/c proceso infeccioso e/p taquicardia, piel caliente rubor, aumento de la temperatura por encima de lo normal $\mathrm{T}: 38.7^{\circ} \mathrm{C}$. Dominio 11, Clase 6.

-(00031) Limpieza ineficaz de las vías aéreas r/c retención de las secreciones $\mathrm{e} / \mathrm{p}$ tos inefectiva, agitación y cambios en la frecuencia respiratoria. Dominio 11, Clase 2.

-(00146) Ansiedad r/c desconocimiento del ambiente hospitalario e/p inquietud, irritabilidad, verbalizaciones de fastidio y llanto. Dominio 9, Clase 2.

\section{FASE DE PLANIFICACIÓN}

Para la realización del plan de atención de enfermería se identificaron los aspectos negativos de la valoración para determinar los diagnósticos de enfermería. Asimismo se seleccionaron las intervenciones de enfermería (NIC) y el NOC respecto al estado respiratorio y al aspecto emocional de la paciente (ver tabla 2).

\section{FASE DE EJECUCIÓN}

Durante los primeros días de hospitalización de la preescolar se ejecutó el plan de intervenciones de enfermería orientado a la recuperación del estado fisiológico principalmente de la respiración y la temperatura corporal. Por otro lado se disminuyó la ansiedad de la niña con apoyo de la madre para que pudiese adaptarse al entorno hospitalario. 


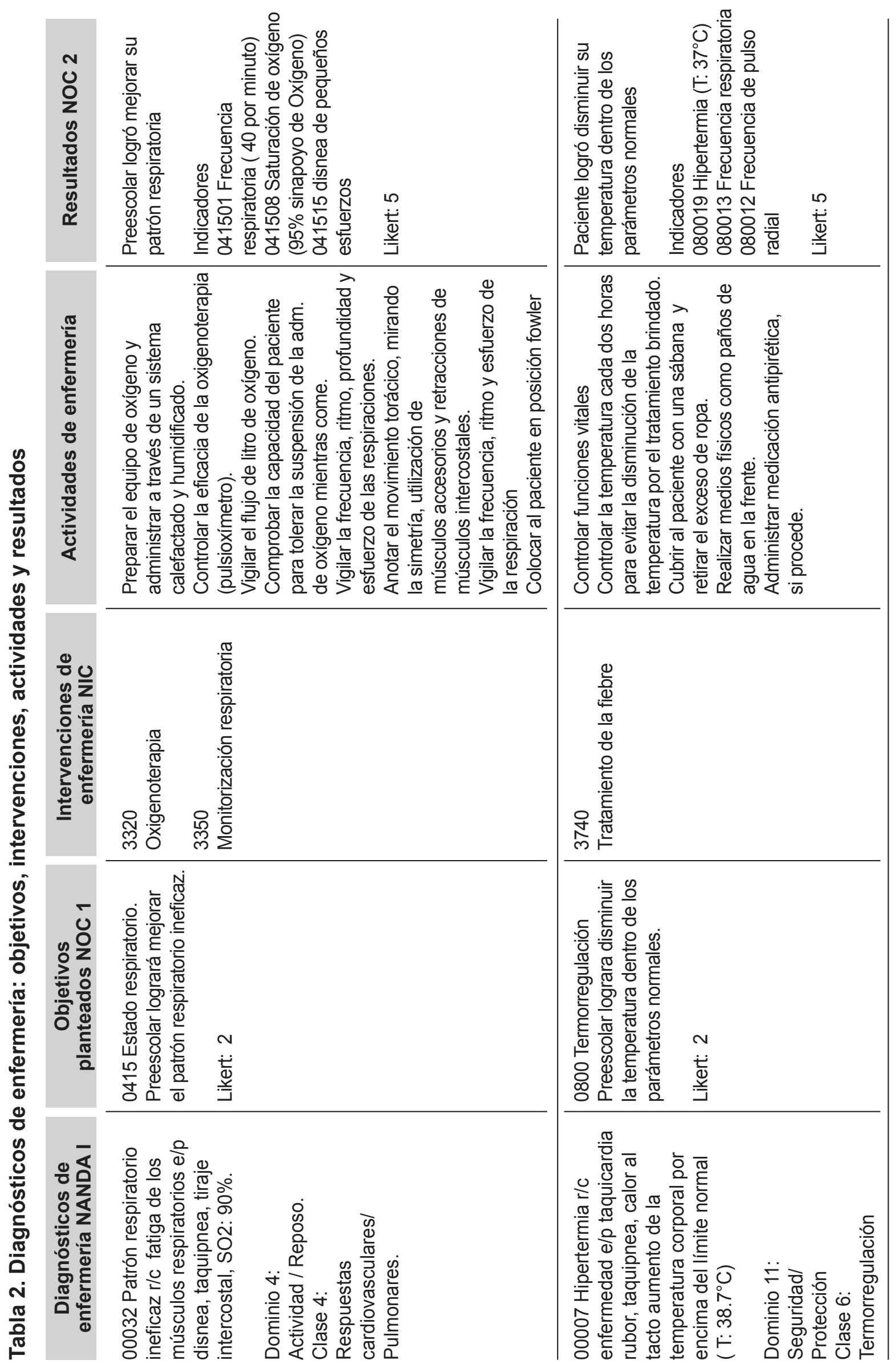




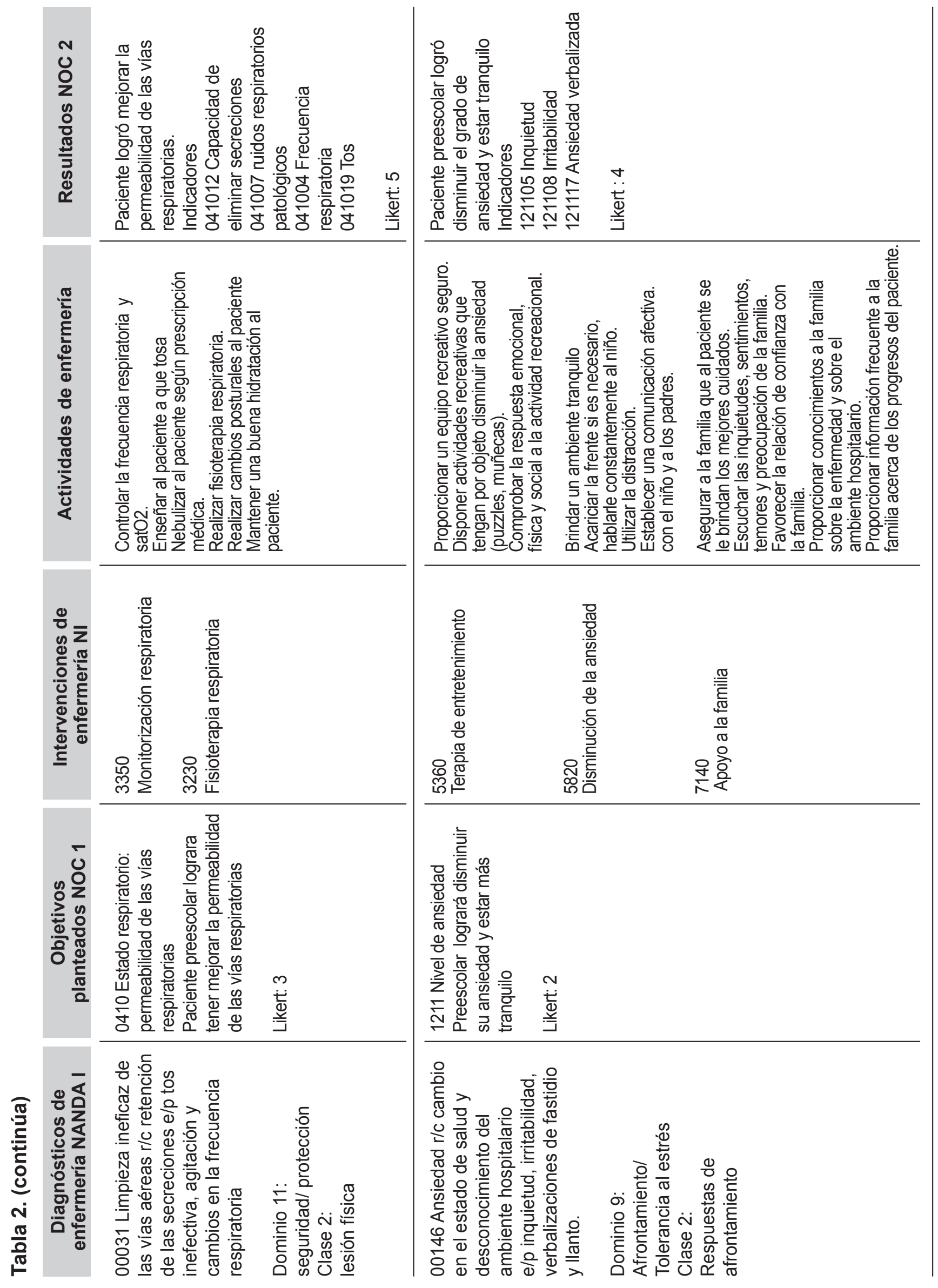




\section{FASE DE EVALUACIÓN}

En la tabla 2 se observa una mejoría notable y positiva en el caso. En el diagnóstico de Patrón respiratorio ineficaz se consiguió alcanzar el objetivo planteado mediante las intervenciones de oxigenoterapia y monitorización respiratoria. La preescolar mejoró su estado respiratorio y por ende el intercambio gaseoso pasando del indicador 2 (desviación sustancial del patrón respiratorio) al indicador 5 (sin desviación del rango normal). Se corroboró mediante la toma de las respiraciones por minuto y la saturación de oxígeno sin apoyo ventilatorio de la cánula binasal comparándolos con los valores normales en preescolares.

En el diagnóstico de Hipertermia se logró con la aplicación del control de la temperatura, medios físicos y el manejo de medicación antipirética para poder disminuir la fiebre. Después de los cuidados de enfermería la paciente alcanzó reducir la temperatura de $38.7{ }^{\circ} \mathrm{C}$ a $37{ }^{\circ} \mathrm{C}$ pasando de un indicador 2 (desviación sustancial de la termorregulación) a un indicador 5 (desviación no comprometida de la termorregulación).

Se priorizó el diagnóstico de Limpieza ineficaz de las vías aéreas debido a la retención de secreciones, tos inefectiva y agitación. Mediante las intervenciones de enfermería fisioterapia respiratoria y monitorización se logró que las secreciones sean removidas a través de la tos y de las nebulizaciones. Se incrementó dos puntos del indicador inicial logrando alcanzar el indicador 5 (sin desviación del rango normal en el estado respiratorio: permeabilidad de las vías respiratorias).

Respecto al diagnóstico de enfermería ansiedad debido al miedo, irritabilidad y llanto por parte de la preescolar se ejecutaron las intervenciones terapia de entretenimiento, disminución de la ansiedad y apoyo a la familia. El indicador inicial mejoró dos puntos y se obtuvo como resultado final 4 (levemente comprometido referente al nivel de ansiedad).

\section{DISCUSIÓN}

Ante la evaluación del plan de atención de enfermería se concluye que se logró mejorar el aspecto fisiológico: patrón respiratorio, la temperatura corporal, la limpieza ineficaz de las vías aéreas y también la ansiedad de la preescolar con el fin de disminuir complicaciones en el estado de salud y psicosocial de la preescolar. Asimismo, se consiguió restablecer el patrón respiratorio de la paciente logrando un buen estado respiratorio y con una saturación de oxígeno dentro de los parámetros normales.

En un estudio se demostró la efectividad de las intervenciones de enfermería a la preescolar con neumonía. La oxigenoterapia es la intervención terapéutica más aconsejable para el tratamiento de las alteraciones de la ventilación, perfusión alveolar e hipoxia. La misma ayuda a conservar los niveles de oxígeno adecuados en el ser humano a través de la captación y transporte y una adecuada ventilación perfusión alveolar (4). Asimismo la monitorización respiratoria es esencial en la atención del infante. $\mathrm{Su}$ empleo y la adecuada interpretación de los valores respiratorios permiten intervenciones en las alteraciones respiratorias (6).

Respecto al manejo de la temperatura corporal un estudio sobre el tratamiento de la fiebre evidencia que la combinación de administración de antipiréticos e intervenciones por medios físicos como el retirar la ropa del niño, poner paños tibios en la frente, aumentar el consumo de líquidos, brindar un ambiente templado y ventilado disminuyen la temperatura corporal en niños de manera efectiva (12).

La fisioterapia y la nebulización son procedimientos efectivos que ayudaron a mejorar el estado respiratorio de la preescolar conjuntamente con los otros síntomas relacionados. La literatura confirma la importancia de la nebulización como vía de administración de medicamentos que favorecen la eliminación de secreciones $(13,14)$. Asimismo la fisioterapia respiratoria es efectiva para eliminar las secreciones con el fin de mejorar la saturación de oxígeno del paciente durante y después de haberla recibido (7).

Por otro lado, la ansiedad del niño consecuente a la estancia hospitalaria ocasiona emociones y conductas de temor, inquietud y miedo. Por esta razón, es necesario realizar estrategias que favorezcan la disminución de la ansiedad y el estrés, tanto del menor como de los padres. Las mismas tienen la finalidad de ayudar en los cuidados del paciente y enseñarles a los padres a enfrentar su hospitalización (8). Por lo cual, se realizaron diversas actividades como la terapia de entretenimiento haciendo que la creatividad del niño fluya a través de la imaginación de ambientes, panoramas logrando que su atención no 
se centre en el hecho de estar hospitalizado (8). Otras intervenciones que se realizaron fueron los juegos, ejercicios de relajación y la comunicación entre la enfermera y el paciente $(8,15)$. Es imprescindible en la atención al paciente involucrar a los padres o cuidadores. Por esta razón es necesario establecer una comunicación con los padres con el fin de orientar y enseñar sobre las causas, síntomas, tratamiento y evolución de la enfermedad. Igualmente se les debe brindar apoyo emocional que les ayude a hacer capaces de afrontar la enfermedad del menor. De este modo se convierten en un apoyo valioso para el personal de salud en la recuperación de sus hijos (15).

Las dificultades que se presentaron al momento de realizar los cuidados estuvieron vinculadas al hacinamiento en el servicio de pediatría lo cual impedía dedicar el tiempo adecuado a las intervenciones propuestas en el plan de cuidados. Asimismo este factor afectó igualmente el tiempo que se tuvo para educar a los padres en relación a la prevención y cuidados de la enfermedad.
Se recomienda que los profesionales de enfermería realicen una monitorización respiratoria continua. Por otro lado se sugiere comunicar a los padres con el fin de educar y orientar sobre el estado de salud del niño. Siendo una comunicación de confianza en términos comprensibles y en un ambiente propicio que garantice la intimidad y la confidencialidad. En los diferentes establecimientos de salud, también se recomienda aplicar los planes de cuidados de enfermería basados en la taxonomía NANDA, NIC y NOC. Estos son de gran ayuda para evaluar las intervenciones realizadas en el paciente y la efectividad de las mismas. Ante la evaluación se concluyó que la paciente logró recuperar su estado respiratorio, recibiendo el alta a los tres días de hospitalización con una frecuencia respiratoria de 40 por minuto, $\mathrm{SatO} 2>95 \%$ sin apoyo de oxígeno, $\sin$ presencia de tirajes intercostales y ausencia de secreciones en ambos campos pulmonares.

\section{REFERENCIAS BIBLIOGRÁFICAS}

1. WHO. Neumonía. Nota descriptiva [Internet]. Ginebra: OMS; 2016 [citado el 22 febrero de 2017]. Disponible en:

http://www.who.int/mediacentre/fact sheets/fs331/es/.

2. Ruuskanen O, Mertsola J, Childhood community-acquired pneumonia. Semin Respir Infect. 1999;14:163-72.

3. Yon C. Situación epidemiológica de las infecciones respiratorias agudas (IRA), neumonías y SOB (asma) en el Perú hasta la SE 17. Boletín Epidemiológico.

2015;24(17):339-340.

4. Rodríguez Ibagué LF, Díaz Castillo LÁ, Martínez Santa J Oxigenoterapia. Primera edición. Bogotá: Editorial Universidad del Rosario; 2008.

5. Botella Dorta C. Oxigenoterapia: Administración en Situaciones de Hipoxia Aguda. C.S La Laguna -Mercedes .Servicio Canario de la Salud. Islas Canarias, España .2005. [Artículo de Investigación publicado en la internet].Disponible en: https://www.fisterra.com/mateial/tec nicas/oxigenoterapia/oxigen2.pdf Fecha de revisión 7 de Marzo 2017.
6. Donoso A, Arraigada D, Contreras D, Ulloa D, Neumann M. Monitorización Respiratoria del Paciente Pediátrico en la Unidad de Cuidados Intensivos. Santiago-Chile: Hospital Clínico Metropolitano La Florida. Bol Med Hosp Infant Mex .

2016;73(3):149-165.

7. Gonzales Bellido V, Gonzales Olmedo P, Martínez Martínez A. Neumonías de Repetición y Fisioterapia Respiratoria: a propósito de un Caso Clínico. Madrid, España: Departamento de Fisioterapia, Universidad Francisco de Vitoria, Pozuelo de Alarcón. Fisioterapia 2009;31(1):32-35.

8. Méndez F, Ortigosa JM, Pedroche S. Preparación a la Hospitalización Infantil (1): Afrontamiento al Estrés. Murcia, España. Universidad de Murcia. Facultad de Psicología. Revista Psicología Conductual 1996;4 (2)193-209.

http://www.behavioralpsycho.com/P DFespanol/1996/art03.2.04.pdf

9. Herdman TH, Kamitsuru S. NANDA International . Diagnósticos Enfermeros: Definiciones y Clasificación, 2015-2017. Barcelona: Elsevier; 2015.
10. Bulechek GM, Butcher $\mathrm{H}$ K, Dochterman JM, Wagner CM. Clasificación de Intervenciones de Enfermería (NIC). 6ta ed. Barcelona: Elsevier; 2014.

11. Moorhead S, Johnson M, Maas ML, Swanson E. Clasificación de Resultados de Enfermería (NOC). 5ta ed. Barcelona: Elsevier; 2014.

12. Olivia Martínez M, Remigio $R$, Pérez G. La Fiebre un Problema Permanente para el Niño, La Familia y el Profesional de Salud. Revista de Ciencias Médicas La Habana 2005;11(1):161-173.

13. Maiz Carro L, Wagner Struwing C. Beneficios de la Terapia Nebulizada: Conceptos Básicos. Madrid, España. Arch Bronconeumol. 2011;47(6):2-7.

14. Benito Fernández J. Tratamiento con Broncodilatadores en urgencias de pediatría: Nebulización versus Inhalación con Cámara Espaciadora. España. Arch Pediatr Urug 2003;74(2)114-118.

15. Serradas Fonseca M. La Pedagogía Hospitalaria y el Niño Enfermo: Un Aspecto más en la Intervención Socio-Familiar. Caracas: Facultad de Ciencias de la Educación de la Universidad de Carabobo. Rev. Ped. 2003;71(24). 


\section{Nursing care for preschooler with viral pneumonia}

ABSTRACT: Pneumonia is the main cause of morbidity and mortality in children under five years of age. This study presents the case of a two-year-old preschooler who presented respiratory distress, ventilating with oxygen support by Three liters binaural cannula with a $\mathrm{FiO} 2$ of $32 \%$ with $\mathrm{SatO} 2$ 99\%, respiratory rate of 48 per minute, intercostal drawing, presence of rales to auscultation in both lung fields, hot skin and blushing cheeks with $38.7^{\circ} \mathrm{C}$. After the assessment of the case, nursing care planning was carried out. Four real diagnoses were presented: "Ineffective respiratory pattern", "Hyperthermia", "Inefficient airway cleaning" and "Child anxiety". The care plan was carried out during the first days of preschool hospitalization using the 13 domain assessment model, selecting the positive and negative aspects of NANDA-I Taxonomy II and the classification of diagnoses, interventions and results of the Taxonomy II. It was concluded that through the nursing care plan the recovery of the respiratory pattern, hyperthermia and inefficient cleaning of the airways was achieved, and the child's anxiety was also reduced.

KEY WORDS:

Pneumonia viral; Monitoring physiologic;

Oxygen Inhalation Therapy; Anxiety 